\title{
Study on the Free Bending of Metal Sheets Based on the Model of Modified Indices
}

\author{
Jing Tao ${ }^{1, *}$, and Zhengwei $\mathrm{Fu}^{2}$ and Han $\mathrm{Gao}^{3}$ \\ ${ }^{I}$ School of Mechanical and Electrical Engineering, Hubei Polytechnic University, Huangshi 435003, China \\ ${ }^{2}$ School of Machinery and Automation, Wuhan University of Science and Technology, Wuhan 430081, China \\ ${ }^{3}$ Lyle School of Engineering, Southern Methodist University, Dallas, Texas 75206, United States
}

Received 21 June 2020; Accepted 4 August 2020

\begin{abstract}
In the free bending process for sheet metals, determining the holes of lower dies can be difficult due to the different thicknesses of metal sheets, which has a considerable effect on the forming quality of the bent metal sheets. An improved mathematical model of modified indices was proposed in this study to reveal the relationship among metal sheet thickness, lower die hole, and bending angle at the time of free bending and to identify the optimal hole of the lower die for sheets with different thickness during free bending. First, relevant data were obtained through field experiments and processed using a three-point moving average. Second, the data were divided into the "growth period" and the "saturation period" according to the different changing rules of the experimental data. The dimensional model and the model of the modified indices were employed to establish the piecewise fitting modelling. The influence law of the ratio of the lower die hole to sheet thickness on the quality of free bending was also obtained. Finally, ABAQUS was employed to verify the proposed model, and the ratio range of lower die hole to sheet thickness for the optimal quality of free bending was given. Results show that the fitting degree between the influence function of the free bending forming quality established by the improved mathematical model of modified indices and the actual data is $99.8 \%$ while the ratio of lower die hole to sheet thickness for the optimal bending quality of free bending is 7-9. This study provides a certain reference for the selection of the lower die hole for the free bending of metal sheets with different thicknesses.
\end{abstract}

Keywords: Free bending, Modified indices, Bending springback, Forming quality

\section{Introduction}

Among the methods for forming metal sheets, free bending has been widely applied in the modern manufacturing industry with its advantages of low cost, high efficiency, and flexible processing. Springback will inevitably occur during the free bending of metal sheets (hereinafter referred to as "sheets"). Quality problems can be observed in metal sheets with different thicknesses, which are freely bent, including excessive springback and cracking, because of the unreasonable design of the lower die hole [2]. Usually, the hole of the lower die is created based on the experience of the workers during the free bending of sheets with different thicknesses, resulting in the poor bending forming quality of sheets and a waste of resources and manpower.

Therefore, a considerable number of studies have been conducted on the influence factors of free bending [3-5], and the influence rules of different factors on the bending forming quality have been determined, thereby resulting in an improvement in bending forming quality. Through a large number of experimental observations, the bending springback has been found to be inversely proportional to the hole of the lower die and is directly proportional to the thickness of the sheet [3-4]. Hence, the aim is to obtain the rules of material deformation from the perspective of material deformation [5]. However, many factors coupled with one another affect the bending of metal sheets and

*E-mail address: taojing605119917@163.com

ISSN: 1791-2377 @ 2020 School of Science, IHU. All rights reserved. doi:10.25103/jestr.134.22 improving the forming quality of metal sheets by considering only one factor is not very effective. Therefore, identifying the method for selecting the lower die hole to reduce bending springback and improve bending quality has become an urgent problem that needs to be addressed for free bending of metal sheets.

For this purpose, in this study, the function of the effects of the hole of the lower die and the thickness of the sheet on the forming quality of free bending was established using data of the orthogonal test with the dimensional model. The model of modified indices and the rules of influence of the thickness of the sheet and the hole of the lower die on the quality of free bending were analyzed to identify the optimal hole of the lower die for sheets with different thicknesses during free bending and improving the bending quality.

\section{State of the art}

A considerable number of studies have been done by scholars from China and other countries to improve the bending quality of metal sheets. Webb [6] et al. applied the method of the transfer function to obtain the modification amount of the bent die and improved the bending quality by changing the shape of the die. However, local information can be lost easily when the method of the transfer function is used. K. Yilamu [7] et al. analyzed the forming process of bent sheets through experiments and finite elements and studied the effects of sheet thickness on bending springback. However, this method ignored the actual process and failed to provide the relevant mathematical model. Vitalii Vorkov 
[8] et al. employed two different regression fitting methods to explore the characteristics of factors related to the springback of large radius free bending but failed to provide substantive solutions that could improve bending quality. Jamli M R [9] et al. simulated the forming process of metal sheets at the time of bending by embedding the model of the neural network into the software of finite elements. Although the bending forming process of sheets was very well reflected through this method, some errors between the actual production process and the simulation results were still observed. Ponticelli GS [10] et al. attempted to optimize the bending parameters with the fuzzy model to improve bending accuracy. Many factors affect bending, the application of this method is not very effective. Sara S Miranda [11] et al. applied a variety of learning models together with finite element analysis and considered the forming and springback processes separately to obtain the bending process parameters and bent-die model. Although various factors in the bending process have been considered applying this method, the process is more complicated and incurs higher costs. Jyhwen Wang [12] et al. predicted the position of the bending punch by applying the over-bending compensation principle to obtain the expected bending angle. However, the accuracy of this method still needs to be improved, and the model needs to be re-established for the bending of different materials. Through numerical simulation and experiment, Mohamadi S V [13] et al. verified the effects of the punch radius, lower dies hole, and punch stroke on bending and determined that the lower die hole had the greatest effect on bending springback. Under this method, the corresponding quantitative model was not given though many assumptions were applied. Fu Zemin [14] established a model of bending springback radius with the method of dimensional analysis. By applying this model, the nonlinear relationship between the parameters and the bending springback radius can be obtained quantitatively along with the design parameters of the terrace dies. However, the application of this model is subject to the limitations of some parameters. Kongyan [15] et al. obtained the optimal combination of bending process parameters by applying the grey system theory and the method of average correlation degree. This method is applied for optimization within the scope of the experimental data, and thus, is not a universal method. Zhang Xueguang [16] et al. analyzed the effects of material mechanical properties on bending forming through the comparison of the results of the tensile test and finite element model. However, under this method, only qualitative analysis can be carried out. Han Fei [17] et al. optimized neural network with the method of genetic algorithm and established a model of springback prediction for the incremental forming of sheet bending, which can reflect very well the relationship between process parameters and bending springback. However, the interaction rules of various parameters need to be improved under this method. Han Jiang [18] et al. established the model of pre-unloading downward pressure of the upper die of V-shaped free bending based on the function of a radial basis and verified the model through experiment and simulation. However, the actual effect of this model is not ideal. For the effects of the different materials on bending quality, Song Y [19] et al. studied the effects of the materials on springback with the method of the neural network but ignored several factors in the modeling process. Li Ming [20] et al. obtained the rules on the effects of different parameters on bending quality through the quasi-static module of ANSYS Workbench. However, this model involves many simplifications in its establishment that were divorced from actual data, and thus, the effectiveness of the model needs to be verified further.

In most of the above studies, the simulation method was applied to study the bending forming process or the prediction method was used to reduce bending springback. Few studies focused on enhancing the bending quality by improving the relevant factors of the bending model and fewer studies still focused on the selection of the appropriate lower die holes for different sheet thickness. Hence, to address the shortcomings of relevant studies, the model of modified indices was applied in this study. The good fitting of the proposed model was employed to model the bending forming angle, sheet thickness, and lower die hole. Based on the experimental data of free bending of different sheet thickness under the proposed model, the mathematical model of sheet thickness, lower die hole, and bending angle at the time of free bending was established to obtain the optimal value of lower die hole at the time of the optimal bending quality under different sheet thickness and provide the basis for the optimization and experiment of the bending quality of metal sheets.

The rest of this study is structured as follows. In the third section, the function of the effects of the lower die hole and sheet thickness on the forming quality of free bending is established by using the orthogonal test of free bending and applying the model of modified indices according to the experimental data. In the fourth section, the established model of modified indices of free bending is verified and validated through simulation using ABAQUS. In the last section, the whole study is summarized, and the relevant conclusions are given.

\section{Methodology}

\subsection{Model of modified indices}

The model of modified indices is employed to simulate the growth process of the biological population, which can fit the morphological curves of various indices very well; this mathematical model can also reflect very well the rules of the change of things [21-22]. At the time of sheet bending, sheet thickness and lower die hole have considerable effects on bending quality, roughly taking on different index forms as a rule. The model of modified indices was employed to establish the mathematical model of sheet thickness, lower die hole, and bending springback and identify the appropriate hole of the lower die for free bending under different sheet thicknesses. The initial sequence is expressed by Formula (1).

$$
X(t)=\{x(1), x(2), x(3) \cdots x(n) \cdots\}
$$

The basic forms of the model of modified indices can be expressed by Formula (2):

$x(t)=k+a b^{t}$

where, $k, a$, and $b$ are parameters, $t$ is an independent variable of time and $x(t)$ is a dependent variable.

The parameters were solved according to the three-sum method. Three groups of data were selected from the initial sequence $x(t)$, with each group containing $n$ initial sequences of bending with the same number. The spacing of the selected initial sequence is equal, namely $(t+1)-t=n$. The sums of the three groups of data are as follows: 


$$
\begin{aligned}
& \sum_{1} x_{t}=\sum_{t=1}^{n} x_{t}=n k+a b\left(b^{0}+b^{1}+\cdots+b^{n-1}\right) \\
& \sum_{2} x_{t}=\sum_{t=n+1}^{2 n} x_{t}=n k+a b^{n+1}\left(b^{0}+b^{1}+\cdots+b^{n-1}\right) \\
& \sum_{3} x_{t}=\sum_{t=2 n+1}^{3 n} x_{t}=n k+a b^{2 n+1}\left(b^{0}+b^{1}+\cdots+b^{n-1}\right)
\end{aligned}
$$

Where $\sum_{1} x_{t}, \sum_{2} x_{t}$, and $\sum_{3} x_{t}$ correspond to the sums of various parts of the initial sequence $x(t)$ in the three groups of data.

According to the summation formula of equal ratio sequence, Formula (3) was simplified into Formula (4).

$$
\begin{aligned}
& \sum_{1} x_{t}=n k+a b \frac{b^{n}-1}{b-1} \\
& \sum_{2} x_{t}=n k+a b^{n+1} \frac{b^{n}-1}{b-1} . \\
& \sum_{3} x_{t}=n k+a b^{2 n+1} \frac{b^{n}-1}{b-1}
\end{aligned}
$$

The initial sequence $x(t)$ was brought into Formula (4) to solve the parameter values of $k, a$, and $b$, as shown in Formula (5).

$$
\begin{aligned}
& b=\sqrt[n]{\frac{\sum_{3} x_{t}-\sum_{2} x_{t}}{\sum_{2} x_{t}-\sum_{1} x_{t}}} \\
& a=\left(\sum_{2} x_{t}-\sum_{1} x_{t}\right) \frac{b-1}{\left(b^{n}-1\right)^{2}} . \\
& k=\frac{\sum_{1} x_{t}-\left(\frac{b^{n}-1}{b-1}\right) a}{n}
\end{aligned}
$$

\subsection{Model improvement}

In the process of modeling, the random errors in the measurement and selection of the original data $x(t)$ cause the initial sequence $x(t)$ selected in the modeling process to be rough, resulting in the deviation of the established model. In this study, a three-point moving average was introduced for treatment to improve the model, as shown in Formula (6) to reduce the effects of random errors in the data and improve the fitting accuracy of the model.

$$
\begin{aligned}
& x(1)=\frac{(3 x(1)+x(2))}{4} \\
& x(t)=\frac{(x(t-1)+2 x(t)+x(t+1))}{4} \\
& x(m)=\frac{(x(m-1)+3 x(m))}{4}
\end{aligned}
$$

Where $x(1)$ is the moving average of initial data, $x(t)$ is the moving average of median data, and $x(m)$ is the moving average of final data.

The three-point moving average in Formula (6) was employed to replace the value of $x(n)$ in the initial sequence. The value is associated with its adjacent numerical information at the time of moving average and thus, the value was weighted according to the difference of distance.
In this way, the effects of the random or human error in the original initial sequence on the model were reduced and the smoothness of the model and the validity of the data improved.

\subsection{Collection of data}

Experimental data were collected on the bending machine. A Q235 rolled steel with an overall dimension of $60 \times 20 \mathrm{~mm}$, a lower die hole angle of $78^{\circ}$, an upper-die rounded angle of $0.6 \mathrm{~mm}$, and a lower die rounded angle of $0.8 \mathrm{~mm}$ was used to control the interference of other variables on the experiment. The bending angle before springback was kept at $90^{\circ}$, with the sheet thickness at $1,2,3$, and $4 \mathrm{~mm}$, and the sizes of lower die holes at 20,25,30, and $40 \mathrm{~mm}$. The field test is shown in Figure 1.

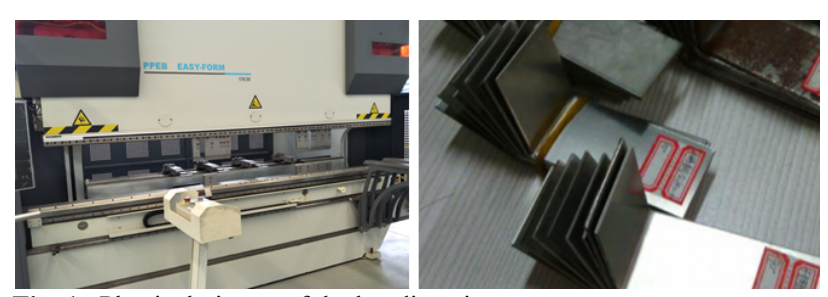

Fig. 1. Physical picture of the bending site

To reduce the frequency of the experiment, the preceding two items of the orthogonal table $L_{16}\left(4^{5}\right)$ were selected for a test. Data on the bending forming angles in the test are shown in Table 1.

Table. 1. Orthogonal test table of free bending

\begin{tabular}{c|c|c|c|c}
\hline $\begin{array}{c}\text { Frequency } \\
\text { of test }\end{array}$ & $\begin{array}{c}\text { Sheet } \\
\text { thickness }(d)\end{array}$ & $\begin{array}{c}\text { Hole } \\
(h)\end{array}$ & $\begin{array}{c}\text { Sheet thickness } \\
\text { /hole }(d / h)\end{array}$ & $\begin{array}{c}\text { Forming angle } \\
\left(\theta /{ }^{\circ}\right)\end{array}$ \\
\hline 1 & 1 & 20 & 20 & 98.5 \\
2 & 1 & 25 & 25 & 99.3 \\
3 & 1 & 30 & 30 & 99.9 \\
4 & 1 & 35 & 35 & 100.3 \\
5 & 2 & 20 & 10 & 93.4 \\
6 & 2 & 25 & 12.5 & 94.5 \\
7 & 2 & 30 & 15 & 95.1 \\
8 & 2 & 35 & 17.5 & 97.2 \\
9 & 3 & 20 & 6.7 & 92.7 \\
10 & 3 & 25 & 8.3 & 92.8 \\
11 & 3 & 30 & 10 & 93.2 \\
12 & 3 & 35 & 11.7 & 93.7 \\
13 & 4 & 20 & 5 & 92.4 \\
14 & 4 & 25 & 6.25 & 92.6 \\
15 & 4 & 30 & 7.5 & 92.5 \\
16 & 4 & 35 & 8.75 & 92.9 \\
\hline
\end{tabular}

\subsection{Establishment of the model of modified indices}

The data Table 1 are arranged in the order of the value of $\frac{h}{d}$ (from small to big). To eliminate the random error arising from the collection of data and enhancing the smoothness of data, the treatment of the three-point moving average was performed for the forming angle $\theta$ according to Formula (3). The initial sequence $x(t)$ built after the three-point moving average treatment of the original data to form angle $\theta$ in Table 1 is shown in Table 2.

Table 2. Initial sequence of free bending

\begin{tabular}{c|c|c|c|c|c|c|c|c}
\hline$t$ & 5 & 6.25 & 6.67 & 7.5 & 8.33 & 8.75 & 10 & 10 \\
\hline$\theta /^{\circ}$ & 92.9 & 93.1 & 93.2 & 93 & 93.3 & 93.4 & 93.6 & 93.4 \\
$x(t)$ & 92.95 & 93.075 & 93.125 & 93.125 & 93.25 & 93.425 & 93.5 & 93.525
\end{tabular}




\begin{tabular}{c|c|c|c|c|c|c|c|c|}
$t$ & 11.67 & 12.5 & 15 & 17.5 & 20 & 25 & 30 & 35 \\
$\theta^{\circ}$ & 93.7 & 94.1 & 95.1 & 97.2 & 98.5 & 99.3 & 99.9 & 100.3 \\
$x(t)$ & 93.725 & 94.25 & 95.375 & 97 & 98.375 & 99.25 & 99.85 & 100.2 \\
\hline
\end{tabular}

The line chart of the initial sequence $x(t)$ of the bending forming angles was established according to Table 2 and shown in Fig 2.

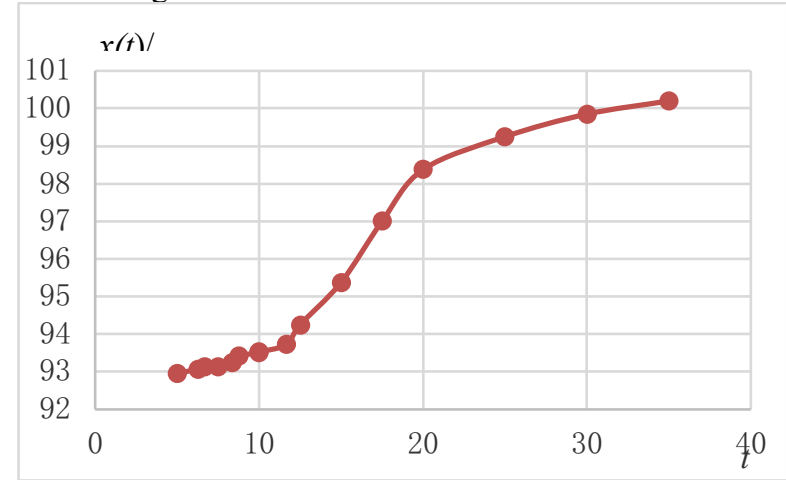

Fig. 2. Chart of the initial sequence of free bending

The improved model of modified indices was employed to fit the initial sequence of free bending to improve the quality of free bending and obtain the optimal hole of lower die for different sheet thickness and the relationship among sheet thickness $(d)$, lower die hole $(h)$, and bending angle $(\theta)$ was determined. However, because the modified indices were only applied for the establishment of the model of two unknown variables, the dimensional homogeneity principle [23] was introduced to establish the mathematical dimensional model related to sheet thickness $(d)$, lower die hole $(h)$, and bending angle $(\theta)$, as shown in Formula (7).

$$
\theta=f \frac{h}{d}
$$

In the formula, $f$ is an unknown function.

Given $t=\frac{h}{d}$ and $\theta=x(t)$, the time variable in the model of modified indices was transformed into the ratio model of lower die hole to sheet thickness in free bending, so that the relationship of three unknown variables in the original bending model was changed to be that of two unknown variables, and the bending model was simplified to meet the modeling requirement of modified indices. The initial sequence $x(t)$ of the model of modified indices is shown in Formula (8).

$$
X(t)=\{92.95,93.075,93.123 \cdots 100.2\}
$$

Fig (2) shows that the model of the ratio of lower die hole to sheet thickness and the bending angle at the time of the free bending of sheets roughly assumes the shape of an " $\mathrm{S}$ ". When $t$ ranges from 5 to 15 , the picture exhibits a concave growth, being "the growth period" of the bending model. When $t$ ranges from 15 to 35 , the picture exhibits a convex growth, being "the saturation period" of the bending model. Modified indices were used to model "the growth period" and "saturation period" of the bending model.

When the modified indices were used to fit the "growth period" of the bending model, the number group with an interval of 5 in the initial sequence $x(t)$ was selected, $\mathrm{n}=5$ at this time, and $x(5)=92.95$ was used as an initial value. The corresponding initial sequence of the "growth period" of free bending is shown in Formula (9).
$X_{1}(t)=\{92.95,93.5,95.375\}$

Formula (1) was brought onto the model of modification as an initial sequence for modeling, and the model of modified indices obtained was restored into the bending model. The fitting function of the "growth period" of the bending model is shown in Formula (10).

$$
\hat{x}(t)_{1}=92.7217+0.2283 \times 3.4091^{\left(\frac{t-5}{5}\right)}(0 \leq t \leq 15)
$$

Likewise, when the model of modified indices was used to fit the "saturation period" of free bending, $x(15)=95.375$, $x(25)=99.25$, and $x(35)=100.2$ were selected as samples for above-mentioned modeling, and $n=10$. The corresponding model of the "saturation period" of the free bending is shown in Formula (11).

$$
\hat{x}(t)_{2}=100.4223-5.0473 \times 0.2323^{\frac{(t-15)}{10}}(15 \leq t)
$$

Based on the combination of Formulas (10) and (11), the formula of the function relation between the bending forming angle $\hat{x}(t)$ fit with the model of modified indices and the ratio $t$ of the lower die hole to sheet thickness can be obtained as shown in Formula (12).

$$
\hat{x}(t)=\left\{\begin{array}{l}
92.7217+0.2283 \times 3.4091^{\left(\frac{t-5}{5}\right)}(0 \leq t \leq 15) \\
100.4223-5.0473 \times 0.2323^{\frac{(t-15)}{10}}(15<t)
\end{array}\right.
$$

In Formula (12), the "growth period" and "saturation period" of the bending model are fit with the model of piecewise modeling. The fitting degree between the bending forming angle function $\hat{x}(t)$ established by the model of modified indices and the initial sequence was greatly improved as shown in Fig. 3. The model avoids the fluctuation problem caused by random errors in the original sequence of the model of modified indices and transforms the relationship of the three unknown variables in the original bending model into the relationship between the two unknown variables in the ratio model with the help of the dimension model.

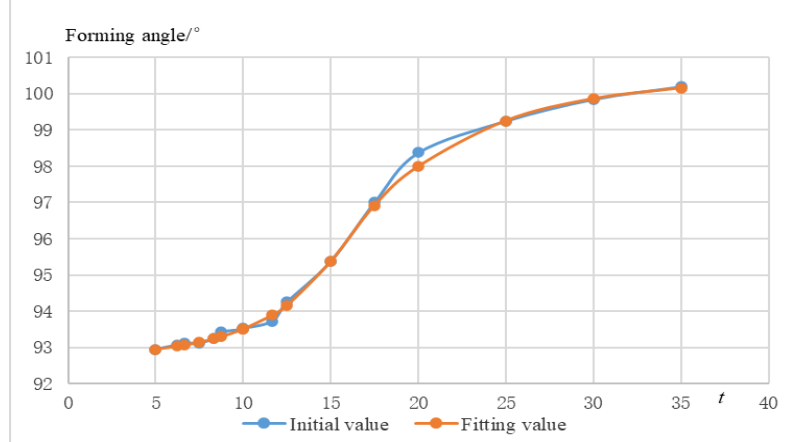

Fig. 3. Fitting of the model of modified indices of free bending

\section{Result Analysis and Discussion}

\subsection{Calibration of model errors}

A relevant model test was carried out using Formula (12) to verify the fitting function established by the model of 
modified indices for the bending forming angle $x(t)$ and the ratio $t$ of lower die hole to sheet thickness.

Test of residual errors:

Following the formula for the calculation of residual errors $e(t)=x(t)-\hat{x}(t)$, the residual error at each point in the model of modified indices and the initial sequence were calculated in turn as shown in Table (3).

Table 3. Residual errors of the model of modified indices and the initial sequence

\begin{tabular}{c|c|c|c|c|c|c|c|c}
\hline$e(t)$ & 0 & 0.0431 & 0.0597 & -0.0182 & 0.0112 & 0.131 & 0 & 0.025 \\
\hline$e(t)$ & -0.168 & 0.0913 & 0 & 0.0816 & 0.385 & 0 & -0.007 & 0.05 \\
\hline
\end{tabular}

Table 3 shows the maximum residual error of piecewise function fitted by the model of modified indices for free bending is 0.385 , thereby indicating that the model of modified indices fits the initial sequence of free bending very well.

Test of the determination coefficient $R^{2}$ :

Using Formula (13), the determination coefficient of the whole bending fitting function and the initial sequence can be obtained:

$$
R^{2}=1-\frac{\sum[x(t)-\hat{x}(t)]^{2}}{\sum[x(t)]^{2}-\frac{\left[\sum x(t)\right]^{2}}{N}}=0.998
$$

The larger and the closer to 1 the determination coefficient $R^{2}$ is, the stronger the correlation of fitting function and initial sequence, and the higher the fitting accuracy. In this study, the determination coefficient of the piecewise function fitted by the model of modified indices for free bending is 0.998 , which indicates that $99.8 \%$ of the values in the initial sequence can be fitted successfully by the established function of the bending forming angle $\hat{x}(t)$ and the ratio $t$ of lower die hole to sheet thickness.

\subsection{Verification of model simulation}

Function fitting was in the "growth period" and "saturation period" of the forming angle of free bending and the ratio of the hole of the lower die to the thickness of sheet with the dimensional model and the model of modified indices, respectively, based on the experimental data. The corresponding mathematical model was established, as shown in Formula (12). ABAQUS simulation was carried out for the sheet of $5 \mathrm{~mm} 45 \#$ steel being bent to $90^{\circ}$ at different lower die holes to verify the model as shown in Fig. 4. The bending forming angle was measured as shown in Table 4.

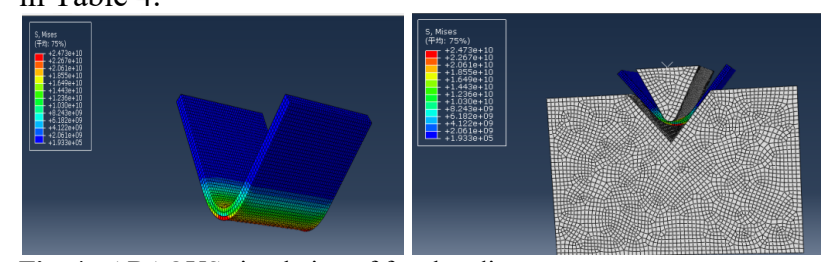

Fig. 4. ABAQUS simulation of free bending

Table 4. ABAQUS simulation data of free bending

\begin{tabular}{c|c|c|c|c|c|c}
\hline$t$ & 1 & 5 & 10 & 15 & 20 & 25 \\
\hline$\theta /{ }^{\circ}$ & Crack & 93.2 (Fissure) & 94.5 & 97.3 & 98.1 & 98.8 \\
\hline
\end{tabular}

Table 4 shows that the smaller the ratio of the hole of the lower die to the thickness of the sheet is, the smaller the springback of bending, which is consistent with the change rules of the established model of modified indices for sheet thickness of free bending, lower die hole and bending angle. Hence, Formula (8) is in line with the rules of actual production. However, when the ratio of the lower die hole to the thickness of the sheet is too small, the sheet will crack or fracture when bent freely. Therefore, to achieve optimal bending quality during free bending, the ratio of lower die hole to sheet thickness should be between 7 and 9. More springbacks will occur with a larger ratio, while more fissures will occur with a smaller ratio.

\section{Conclusions}

To identify the optimal lower die hole at the time of bending of sheets with different thicknesses, the relevant experimental data were obtained through the orthogonal test, while the influence law of the lower die hole and sheet thickness on the forming quality of free bending was established by using the dimensional model and the model of modified indices. The analysis was conducted through model verification and simulation using ABAQUS. The following conclusions can be drawn based on our results:

(1) The improved model of modified indices can reflect very well the relationship between the hole of the lower die, the thickness of the sheet, and the bending angle.

(2) The maximum residual error of the piecewise function of the improved model of modified indices is 0.385 , which fits with the initial sequence of free bending very well.

(3) The smaller the ratio of the hole of the lower die to the thickness of the sheet, the smaller the springback of bending, which is consistent with the change rules of the established model of modified indices for the sheet thickness of free bending, the hole of lower die, and the bending angle. The optimal ratio range is 7-9.

Based on the experimental data and theoretical research, a mathematical model of the effects of lower die hole, sheet thickness, and bending angle on the forming quality of free bending was established. During the free bending of sheets, the proposed model can help in the selection of an appropriate hole in lower die based on the different sheet thicknesses to achieve optimal bending quality. This model could have a high guiding significance to engineering practice. However, because the limited effect of different materials on the proposed model is not considered, in-depth studies on different materials and its related effects in the future.

\section{Acknowledgements}

This work was supported by Hubei Provincial Department of Education Youth talent project of scientific research program (Q20144404)

This is an Open Access article distributed under the terms of the Creative Commons Attribution License 


\section{References}

1. Badr, O. M., Rolfe, B., Zhang P., et al, “Applying a new constitutive model to analyse the springback behaviour of titanium in bending and roll forming”. International Journal of Mechanical Sciences, 128-129, 2017, pp. 389-400.

2. Jamli, M. R., Ariffin, A. K., Wahab, D. A., "Incorporating feedforward neural network within finite element analysis for Lbending springback prediction". Expert Systems with Applications, 42(5), 2015, pp. 2604-2614.

3. Cao, Z. Y., Xia, J. C., Jin, J. S., et al, "Research on metamodel of least square support vector machine for the bending springback". Journal of Plasticity Engineering, 24(2), 2017, pp. 1-9.

4. Li, J. Q., Zhang, S. J., Gong, X. L., et al, "Constitutive parameter identification of complex orthotropic yield functions based on optimization method”. Journal of Plasticity Engineering, 24(1), 2017, pp. 160-167.

5. Gil, I., Mendiguren, J., Galdos, L., et al, "Influence of the pressure dependent coefficient of friction on deep drawing springback predictions". Tribology International, 2016:S0301679X16302225.

6. Webb, R. D., Hardt, D. E., "A transfer function description of sheet metal forming for process control". Transaction of the ASME, Journal of Engineering for Industry, 113, 1991, pp. 44-52.

7. Yilamu, K., Hino, R., Hamasaki, H., Yoshida, F., "Air bending and springback of stainless steel clad aluminum sheet". Journal of Materials Processing Tech, 210(2), 2009, pp. 272-278.

8. Vitalii, V., Richard, A., Dirk, V., Joost, R., Duflou, "Two regression approaches for prediction of large radius air bending". International Journal of Material Forming, 12(3), 2019, pp.379-390.

9. Jamli, M. R., Ariffin, A. K., Wahab, D. A., "Incorporating feedforward neural network within finite element analysis for Lbending springback prediction". Expert Systems with Applications, 42(5), 2015, pp. 2604-2614.

10. Ponticelli, G. S., Guarino, S., Giannini, O., et al, "Springback control in laser-assisted bending manufacturing process by using a fuzzy uncertain model". Procedia CIRP, 88, 2020, pp. 491-496.

11. Miranda, S. S., Barbosa, M. R., Santos, A. D., et al, "Forming and springback prediction in press brake air bending combining finite element analysis and neural networks". The Journal of Strain Analysis for Engineering Design, 53(8), 2018, pp. 584-601.

12. Jyhwen, W., Suhas, V., Richard A., et al, "Springback control of sheet metal air bending process". Journal of Manufacturing Processes, 10(1), 2008, pp. 21-27.
13. Mohammadi, S. V., Parsa, M. H., Aghchai, A. Jalali, "Simplified springback prediction in $\mathrm{Al} / \mathrm{PP} / \mathrm{Al}$ sandwich air bending". Journal of Sandwich Structures and Materials, 17(3), 2015, pp. 217-237.

14. Fu, Z. M., Mo, J. H., Chen, W., et al, "Springback Model for Airbending of Sheet Metal Based on Dimensional Analysis Method". Journal of Mechanical Engineering, 46(12), 2010, pp. 53-58.

15. Kong, Y., Xue, K. M., Li, P., Wu, J., "Simulation and Experiment of Optimized Bending Forming of Thick Sheets of High-Strength Steel Based on Orthogonal Test and Grey System Theory”. Journal of Plastic Engineering, 21 (05), 2014, pp. 11-14 + 40.

16. Zhang, X. G., Lu, K. J., He, G. Z., et al, "Material Properties Fluctuation Effects on Bending Springback of High Strength Steel". Aeronautical Manufacturing Technology, 61(18), 2018, pp. 48-52.

17. Han, F., Mo, J. H., Gong, P., "Springback Prediction of Digital Incremental Forming Based on Genetic Neural Network". Journal of Huazhong University of Science and Technology (Natural Science Edition), (01), 2008, pp. 121-124

18. Han, J., Dou, L. L., X, L., et al, "Springback Prediction of Free Bending of Metal Sheets Based on the Approximation Model of Radial Basis Function”. Forging Technology, 044 (006), 2019, pp. 30-35.

19. Song, Y., Yu, Z., "Springback prediction in T-section beam bending process using neural networks and finite element method". Archives of Civil and Mechanical Engineering, 13(2), 2013, pp. 229-241.

20. Ni, M., Qian, Y., Ping, D. L., Shan, J. Y., "A Study on the Influence Factors of the Springback of Metal Sheet Bending". Light Industry Machinery, 34 (03), 2016, pp. 49-51

21. Song, P. F., "Comparative Study on Population Prediction Methods". Master thesis of Anhui University, China, 2013.

22. Li, X. F., "Population Prediction Model Based on Modified Index Curve". Journal of Jingmen Vocational and Technical College, (06), 2006, pp. 85-88

23. Liang, C. B., Cao, Z. J, Chen, Z. T., "Introduction to Dimensional Analysis (Continued 2)". College Physics, 37 (02), 2018, pp. 12-17

24. Wang, X. M., "Dynamic Prediction of Recursive Compensation by Grey Numbers of Identical Dimensions". Journal of Huazhong University of Science and Technology, (04), 1989, pp. 9-16. 